\title{
Editorial: The Role of Primary Motor Cortex as a Marker and Modulator of Pain Control and Emotional-Affective Processing
}

\author{
Jorge Leite ${ }^{1,2,3}$, Sandra Carvalho ${ }^{1,2}$, Linamara R. Battistella ${ }^{4}$, Wolnei Caumo ${ }^{5,6}$ and \\ Felipe Fregni ${ }^{1 *}$ \\ 1 Spaulding Neuromodulation Center, Spaulding Rehabilitation Hospital, Harvard Medical School, Boston, MA, United States, \\ ${ }^{2}$ Neuropsychophysiology Laboratory, Psychology Research Center (CIPsi), School of Psychology (EPsi), University of Minho, \\ Braga, Portugal, ${ }^{3}$ Portucalense Institute for Human Development (INPP), Universidade Portucalense, Porto, Portugal, \\ ${ }^{4}$ Physical and Rehabilitation Medicine Institute, Medical School General Hospital, University of São Paulo, São Paulo, Brazil, \\ ${ }^{5}$ Post-Graduate Program in Medical Sciences, School of Medicine, Universidade Federal do Rio Grande do Sul, Porto \\ Alegre, Brazil, ${ }^{6}$ Laboratory of Pain and Neuromodulation, Universidade Federal do Rio Grande do Sul, Porto Alegre, Brazil
}

Keywords: motor cortex, stimulation, pain, cognition, emotion

\section{Editorial on the Research Topic}

The Role of Primary Motor Cortex as a Marker and Modulator of Pain Control and Emotional-Affective Processing

In the 1940-50's Wilder Penfield and colleagues applied cortical electrical stimulation to patients undergoing epilepsy surgery to define what has become one of the landmarks on neuroscience: a

OPEN ACCESS

Edited by:

Stephane Perrey,

Université de Montpellier, France

Reviewed by:

Stephane Perrey,

Université de Montpellier, France

Yelena Granovsky,

Rambam Health Care Campus, Israel

*Correspondence:

Felipe Fregni

felipe.fregni@gmail.com

Received: 11 March 2017

Accepted: 08 May 2017

Published: 23 May 2017

Citation:

Leite J, Carvalho S, Battistella LR,

Caumo W and Fregni F (2017)

Editorial: The Role of Primary Motor Cortex as a Marker and Modulator of

Pain Control and Emotional-Affective

Processing.

Front. Hum. Neurosci. 11:270.

doi: 10.3389/fnhum.2017.00270 map of the anatomical divisions of the body, divided in two cortical homunculi: sensory and motor (Penfield and Boldrey, 1937).

Ever since, the development of new tools to investigate brain function non-invasively increased knowledge about the structure and functions of the primary motor Cortex (M1) beyond motor control in both humans and animals. For instance, the role of M1 in visuomotor transformations, mental imagery, or mental rotation has been shown in studies dating more than 30 years ago (Georgopoulos and Pellizzer, 1995; Kosslyn et al., 1998). Also, M1 seems to be activated during memory retrieval of sensory information or finger tapping sequences after a short delay (Kaas et al., 2007), suggesting the M1 involvement with memory processes; as well as involved in language processing of action related words (de Lafuente and Romo, 2004; Hauk et al., 2004; Pulvermuller, 2005 for review). Furthermore, the involvement of the M1 region in higher cognitive functions has also been demonstrated in emotional processing. There seems to be a correlation between sensorimotor activation and empathy (Lamm et al., 2007), as well as relationship between sensorimotor activation and emotional processing in silent reading of emotionally laden words (Papeo et al., 2012). Moreover, M1 seems to be asymmetrically modulated by here emotionally laden sounds, with unpleasant sounds resulting in higher facilitation od motor evoked potentials in the left hemisphere, whereas pleasant sounds resulted in higher excitability in the right side (Komeilipoor et al., 2013).

The involvement of the M1 region in higher cognitive functions was also supported by a recent meta-analysis of neuroimaging findings in which an activation likelihood estimation was used to determine topographic convergence (Tomasino and Gremese, 2016). In the meta-analysis, the M1 subregion 4a was commonly activated during motor imagery and working memory, emotion/empathy, and language. But the potential role of M1 in higher cognitive functions is not limited to the activation of specific brain regions during task performance. By understanding how 
M1 modulates distant neural structures and its relationship with respective brain behavior, $\mathrm{M} 1$ can also be used as a potential marker for clinical applications, as well as to guide neuromodulatory therapeutic options (DaSilva et al., 2012; Carvalho et al., 2015). It is well known, for instance, that M1 has connections with several areas of the brain, and the stimulation of the motor cortex can induce changes in other systems (e.g., pain: Fregni et al., 2006; Castillo-Saavedra et al., 2016). Moreover, stimulation of motor cortex may actually improve cognitive functioning by the activation of corticostriatal-thalamo-cortical loops (CSTC) (Leite et al., 2011).

Considering the role of M1 in cognitive functioning that surpass the motor processing, we proposed a research topic about the relationship between M1 and behavior, namely those related to pain and emotional-affective processing. We were interested in both theoretical and empirical contributions related to electrophysiological, pharmacological, neuroimaging, and neuromodulatory studies.

This special topic comprises 15 articles from a diverse group of scientists that provide a robust contribution for the development to the field. We also want to acknowledge the invaluable help that all reviewers provided during this process-many of them leaders in their field-whose contribution improved significantly the manuscripts. The reviews in this special issue investigate the role of motor cortex when using stimulation techniques to M1 to investigate pain modulation (Brasil-Neto) and how noninvasive brain stimulation can be used for reverting abnormal neuroplasticity associated with chronic pain (Naro et al.). This focus of M1 neuromodulation on pain modulation is also the focus of original studies in different types of pain, such as chronic musculoskeletal and post stroke pain, pain related to chemotherapy, fibromyalgia, or neuropathic pain (Botelho et al.; Caumo et al.; Hu et al.; Luu et al.; Mendonca et al.; O'Brien et al.). Additionally, a framework addressing the contralateral inhibition of the impaired hemisphere following stroke and its potential relationship with central post stroke pain is proposed (Morishita and Inoue). A second common theme was the use of EEG to understand changes in M1, and correlate this neural signal with pain and emotional processing in stroke patients (Doruk et al.) and chronic pain secondary to rheumatoid arthritis (Meneses et al.). Furthermore, the use of neuroimaging was also the topic of one study assessing connectivity alterations in

\section{REFERENCES}

Carvalho, S., Goncalves, O. F., Soares, J. M., Sampaio, A., Macedo, F., Fregni, F., et al. (2015). Sustained effects of a neural-based intervention in a refractory case of tourette syndrome. Brain Stimul. 8, 657-659. doi: 10.1016/j.brs.2014.12.008

Castillo-Saavedra, L., Gebodh, N., Bikson, M., Diaz-Cruz, C., Brandao, R., Coutinho, L., et al. (2016). Clinically effective treatment of fibromyalgia pain with high-definition transcranial direct current stimulation: phase II open-label dose optimization. J. Pain 17, 14-26. doi: 10.1016/j.jpain.2015.09.009

DaSilva, A. F., Mendonca, M. E., Zaghi, S., Lopes, M., DosSantos, M. F., Spierings, E. L., et al. (2012). tDCS-induced analgesia and electrical fields in pain-related neural networks in chronic migraine. Headache 52, 1283-1295. doi: 10.1111/j.1526-4610.2012.02141.x patients with rheumatoid arthritis and correlating increased pain perception with increased connectivity for the supplementary motor areas, mid-cingulate cortex, and the primary sensorimotor cortex (Flodin et al.). Finally roles of the motor cortex on other cognitive domains were also explored, namely M1 activation with real or mental imagery (Galdo-Alvarez et al.), kinematic changes associated with pain in patients with fibromyalgia (Costa et al.), or changes in motor cortex activity following observation of emotionally laden pictures (Nogueira-Campos et al.).

This special topic highlights the role of the motor cortex that goes way beyond motor functioning. Also that we need to expand our knowledge about this particular region, its corticocortico and cortico-subcortico interactions, and how it can modulate or be modulated by different bottom-up (such as median nerve stimulation) or top down (such as TMS or tDCS) interventions. Despite that, this special topic clearly emphasizes methods to probe and neuromodulate motor cortex functioning and its potential impact for comprehensive rehabilitation (such as pain). But those are only a few examples of how motor cortex is involved in pain processing and higher order cognitive processing.

\section{AUTHOR CONTRIBUTIONS}

All authors listed, have made substantial, direct and intellectual contribution to the work, and approved it for publication.

\section{FUNDING}

JL and SC are supported by the Portuguese Foundation for Science and Technology (FCT) and European Union (FSEPOPH) with individual awards FRH/BPD/86027/2012) and (IF/00091/2015). JL, SC are members of CIPSi, supported by the Portuguese Foundation for Science and Technology and the Portuguese Ministry of Science, Technology and Higher Education through national funds and co-financed by FEDER through COMPETE2020 under the PT2020 Partnership Agreement (POCI-01-0145-FEDER-007653); and also through the Portuguese Foundation for Science and Technology PTDC/MHC-PCN/3950/2014. FF is funded by the following NIH grants: R21HD079048, R01HD082302, 1R44NS080632-01, 1R44AT008637, HD069776. de Lafuente, V., and Romo, R. (2004). Language abilities of motor cortex. Neuron 41, 178-180. doi: 10.1016/S0896-6273(04)00004-2

Fregni, F., Gimenes, R., Valle, A. C., Ferreira, M. J., Rocha, R. R., Natalle, L., et al. (2006). A randomized, sham-controlled, proof of principle study of transcranial direct current stimulation for the treatment of pain in fibromyalgia. Arthritis Rheum. 54, 3988-3998. doi: 10.1002/art.22195

Georgopoulos, A. P., and Pellizzer, G. (1995). The mental and the neural: psychological and neural studies of mental rotation and memory scanning. Neuropsychologia 33, 1531-1547. doi: 10.1016/0028-3932(95) 00079-I

Hauk, O., Johnsrude, I., and Pulvermuller, F. (2004). Somatotopic representation of action words in human motor and premotor cortex. Neuron 41, 301-307. doi: 10.1016/S0896-6273(03)00838-9 
Kaas, A. L., van Mier, H., and Goebel, R. (2007). The neural correlates of human working memory for haptically explored object orientations. Cereb. Cortex 17, 1637-1649. doi: 10.1093/cercor/ bhl074

Komeilipoor, N., Pizzolato, F., Daffertshofer, A., and Cesari, P. (2013). Excitability of motor cortices as a function of emotional sounds. PLoS ONE 8:e63060. doi: 10.1371/journal.pone.0063060

Kosslyn, S. M., DiGirolamo, G. J., Thompson, W. L., and Alpert, N. M. (1998). Mental rotation of objects versus hands: neural mechanisms revealed by positron emission tomography. Psychophysiology 35, 151-161. doi: 10.1111/1469-8986.3520151

Lamm, C., Nusbaum, H. C., Meltzoff, A. N., and Decety, J. (2007). What are you feeling? Using functional magnetic resonance imaging to assess the modulation of sensory and affective responses during empathy for pain. PLoS ONE 2:e1292. doi: 10.1371/journal.pone.0001292

Leite, J., Carvalho, S., Fregni, F., and Goncalves, O. F. (2011). Taskspecific effects of tDCS-induced cortical excitability changes on cognitive and motor sequence set shifting performance. PLOS ONE 6:e24140. doi: 10.1371/journal.pone.0024140

Papeo, L., Rumiati, R. I., Cecchetto, C., and Tomasino, B. (2012). On-line changing of thinking about words: the effect of cognitive context on neural responses to verb reading. J. Cogn. Neurosci. 24, 2348-2362. doi: 10.1162/jocn_a_ 00291

Penfield, W., and Boldrey, E. (1937). Somatic motor and sensory representation in the cerebral cortex of man as studied by electrical stimulation. Brain 60 , 389-443. doi: 10.1093/brain/60.4.389

Pulvermuller, F. (2005). Brain mechanisms linking language and action. Nat. Rev. Neurosci. 6, 576-582. doi: 10.1038/nrn1706

Tomasino, B., and Gremese, M. (2016). The cognitive side of M1. Front. Hum. Neurosci. 10:298. doi: 10.3389/fnhum.2016.00298

Conflict of Interest Statement: The authors declare that the research was conducted in the absence of any commercial or financial relationships that could be construed as a potential conflict of interest.

Copyright (C) 2017 Leite, Carvalho, Battistella, Caumo and Fregni. This is an openaccess article distributed under the terms of the Creative Commons Attribution License (CC BY). The use, distribution or reproduction in other forums is permitted, provided the original author(s) or licensor are credited and that the original publication in this journal is cited, in accordance with accepted academic practice. No use, distribution or reproduction is permitted which does not comply with these terms. 